\title{
Atoms as rods and clocks in a gravitational field
}

\author{
A.I.Nikishov \\ Lebedev Physical Institute, Moscow
}

14th Lomonosov Conference. 19-25 August 2009 


\section{Abstract}

We consider the implications of using an atom as a rod and a clock in a constant gravitational field. One consequence of this is that the space metric of any constant gravitational field should be locally isotropic. This fixes the privileged coordinate system. In it the deflection of light at finite distances from the Sun is given by the tangent to the trajectory. In general this approach agrees with general relativity only in linear approximation and this is quite surprising. 
Introduction and exposition of the approach

Almost 50 years ago the authors of [1] proposed to use, for example, the hydrogen atom as rod and clock in gravitational field and analyzed the consequences of this, see also [2]. The gravitational field, according to its strength, modifies the Bohr radius $r_{b}$

$$
r_{b} \rightarrow r_{b \phi}=\frac{r_{b}}{\sqrt{g_{s}}}, \quad r_{b}=\frac{\hbar^{2}}{m e^{2}} .
$$

We use the notation

$$
d s^{2}=g_{00} c^{2} d t^{2}+g_{s}\left(d x_{1}^{2}+d x_{2}^{2}+d x_{3}^{2}\right), \quad g_{s} \equiv g_{11}=g_{22}=g_{33} .
$$

Or denoting

$$
g_{\mu \nu}=\eta_{\mu \nu}+h_{\mu \nu}, \quad \eta_{\mu \nu}=\operatorname{diag}(-1,1,1,1,),
$$

we have

$$
h_{s}=h_{11}=h_{22}=h_{33},
$$

$\ln (2) c^{2}$ is the square of velocity of light measured by local rods and clocks. It is constant and we can put it equal to unity. 
So, according to (1) the atom, shrinks:

$$
\Delta I \rightarrow \Delta I_{\phi}=\frac{\Delta I}{\sqrt{g_{s}}}=\Delta /(1+\phi+\cdots) .
$$

But we are unable to notice this change observing the atom locally (near it). To consider the size of the atom as the same anywhere in gravitational field we have to multiply its shrunken size by the correcting factor $\sqrt{g_{s}}$. In this way the space part of the metric (2) appears.

Similarly we treat the period of oscillation $\mathrm{T}$, interval $\Delta t$ and frequency $\omega$ :

$$
\begin{gathered}
T \rightarrow T_{\phi}=\frac{T}{\sqrt{\left|g_{00}\right|}}, \quad \Delta t \rightarrow \Delta t_{\phi}=\frac{\Delta t}{\sqrt{\left|g_{00}\right|}}, \\
\omega \rightarrow \omega_{\phi}=\omega \sqrt{\left|g_{00}\right|}=\omega(1+\phi+\cdots),
\end{gathered}
$$


(It is said that $\omega_{\phi}$ is measured in the world time $t$, i.e. by the clock ticking in the flat space time from which we start to calculate the influence of the gravitational field on the atom.) The appearance of metric (2) becomes evident.

It follows from (2) that the coordinate velocity of light is given by

$$
c_{\phi}^{2}=c^{2} \frac{\left|g_{00}\right|}{g_{s}}=c^{2}(1+4 \phi+\cdots) .
$$

The rest energy $m c^{2}$ in the gravitational field becomes

$$
m c^{2} \rightarrow m_{\phi} c_{\phi}^{2}=m c^{2} \sqrt{\left|g_{00}\right|}=m c^{2}(1+\phi+\cdots),
$$

see, for example, eq. (88.9) in [4]. From (7) and (8) we have

$$
m \rightarrow m_{\phi}=m \frac{g_{s}}{\sqrt{\left|g_{00}\right|}}=m(1-3 \phi+\cdots),
$$

and from (6) and (8) $m c^{2} T=m_{\phi} c_{\phi}^{2} T_{\phi}$, i.e. the quantity of dimension erg $\cdot \mathrm{sec}$ is independent of $g_{\mu \nu}$. It follows from here (and from $\left.\omega T=\omega_{\phi} T_{\phi}, E=\hbar \omega\right)$ that Planck constant $\hbar$ must also be independent of gravitational field [1] and we can put $\hbar=1$. 
The same should be true for dimensionless fine structure $\alpha=\frac{e^{2}}{\hbar c}=\frac{e_{\phi}^{2}}{\hbar c_{\phi}}$. From here

$$
e^{2} \rightarrow e_{\phi}^{2}=e^{2} \frac{c_{\phi}}{c}=e^{2} \sqrt{\frac{\left|g_{00}\right|}{g_{s}}}=e^{2}(1+2 \phi+\cdots) .
$$

As modifications of physical quantities are determined by their dimensions [2] and the terms of the Dirac equation (or any other equation) are of the same dimension, each term acquire the same factor. For this reason we are unable to notice any change in the atom in gravitational field, when observe it locally [3]. This should be true as long as the tidal forces inside the atom are negligible It is interesting to note that the Einstein gravitational constant

$$
\varkappa \rightarrow \varkappa_{\phi}=\frac{\varkappa}{\sqrt{g_{s}\left|g_{00}\right|}}
$$

is unaffected by gravitational field, if

$$
g_{s}\left|g_{00}\right|=1 .
$$


Now, the picture in which we are dealing with quantities with subscript $\phi$ Thirring calls the unrenormalized one. The picture, in which all the quantities have the same value in the gravitational field as outside of it, Thirring calls the renormalized picture.

Using the atom as a rod, we conclude that the space part of metric of a constant gravitational field should be locally isotropic. i.e. have the form (2). In linear approximation we have $g_{00}=-1-2 \phi$, see eq. (87.11) in [4]. Using linearized Einstein equation and Hilbert gauge

$$
\bar{h}_{, \nu}^{\mu \nu} \equiv\left(h^{\mu \nu}-\frac{1}{2} \eta^{\mu \nu} h\right)_{, \nu}=0, \quad h=h_{\sigma}^{\sigma}, \quad h_{, \nu} \equiv \frac{\partial}{\partial x^{\nu}} h,
$$

we find $g_{s}=1-2 \phi \approx 1 /\left|g_{00}\right|$. (We are interested in metric outside the body.) In these equations $\phi$ is the Newtonian potential of a single body of any form and structure. In this linear approximation it is not difficult to take into account the rotation

$$
h_{0 \alpha}=2 G M_{\alpha \beta} \frac{n_{\beta}}{r^{2}},
$$

see eq. (105.16) in [4]. 
Application: deflection of light by the Sun

- In linear approximation the deflection of light by the Sun can be calculated in two different ways. First, following Schwinger ( see§4 Ch 2 in[5]) we can find the angle of deflection by purely mechanical means.

$$
\varphi=r_{g} \rho \int_{-\infty}^{x} \frac{d x}{\left(x^{2}+\rho^{2}\right)^{3 / 2}}=\frac{r_{g}}{\rho}\left(1+\frac{x}{\sqrt{\rho^{2}+\left(x^{2}\right.}}\right)=\left(1 \mp \sqrt{1-u^{2}}\right) \delta,
$$

Here $\rho$ is the impact parameter, $u=\rho / r$. The signs $\mp$ in front of square root in the last equation refers to points on the ingoing and outgoing parts of trajectory (i.e. before and after passing the Sun) Second, we can calculate the tangent to the trajectory

$$
\begin{gathered}
\tan \varphi=\frac{d y}{d \vartheta} / \frac{d x}{d \vartheta}=\frac{\frac{d r}{d \vartheta} \sin \vartheta+r \cos \vartheta}{\frac{d r}{d \vartheta} \cos \vartheta-r \sin \vartheta}, \\
x=r \cos \vartheta, \quad y=r \sin \vartheta,
\end{gathered}
$$


or in terms of $u=\rho / r$ :

$$
\tan \varphi=\frac{\frac{d u}{d \vartheta} \sin \vartheta-u \cos \vartheta}{\frac{d u}{d \vartheta} \cos \vartheta+u \sin \vartheta} .
$$

The trajectory in the privileged (i.e. isotropic) metric in the considered approximation has the form (see eq. (40.6) in [7])

$$
u=\sin \theta+(1-\cos \theta) \delta .
$$

Using it, we get the same result (14).

For the upper sign in (14) for small $u$ we have

$$
\varphi=\left(1-\sqrt{1-u^{2}}\right) \delta=\left(\frac{1}{2} u^{2}+\frac{1}{8} u^{4}+\cdots\right) \delta .
$$

This expression gives also the amount of decrease of the total deflection angle (which can be not small and may be known only experimentally ) on the second half of the trajectory. In other words, the deflection angle at distance $u$ is the total deflection angle minus the expression (14a). 
Using the standard (in Weinberg terminology [6]) Schwarzschild metric

$$
d s^{2}=\left(1-\frac{r_{g}}{r}\right)\left(d x^{0}\right)^{2}-r^{2}\left(\sin ^{2} \theta d \varphi^{2}+d \theta^{2}\right)-\left(1-\frac{r_{g}}{r}\right)^{-1} d r^{2}
$$

it is easy to get instead (16) the trajectory in the form

$$
u=\sin \vartheta+\left(1-\frac{1}{2} u^{2} \mp \sqrt{1-u^{2}}\right) \delta
$$

and for it instead of (14)

$$
\varphi=\left(1 \mp \sqrt{1-u^{2}}\left(1+\frac{u^{2}}{2}\right)\right) \cdot \delta
$$

For the upper sign we have instead of (14a)

$$
\tan \varphi \approx \varphi=\left(1-\sqrt{1-u^{2}}\left(1+\frac{u^{2}}{2}\right)\right) \delta=\frac{3}{8} u^{4} \delta+\cdots .
$$

The difference with (14) and (14a) shows that the standard Schwarzschild coordinate system contains features introduced not only by the gravitational field but also by arbitrariness in choice of coordinate system. 


\section{Conclusions}

As mentioned earlier, it seems reasonable to expect that the considered approach should hold beyond the linear approximation as long as the tidal force inside the atom is negligible. It even seems reasonable to think that two functions $g_{00}$ and $g_{s}$ are functions of only Newtonian potential. This is the case for the isotropic coordinate system of the Schwarzschild field. It is inticing to think that the same form of metric should hold for any single body; only the Newtonian potentials are specific. But this agrees with general relativity only in linear approximation because only two functions $g_{00}$ and $g_{s}$ mast satisfy all Einstein equations. It would be also interesting to check experimentally that the relation (11a) holds only in linear approximation as predicted by general relativity. 


\section{References}

1. H. Dehnen, H. Hönl, and K. Westpfahl, Ann. Phys. (Leipzig) 461, 370 (1960).

2. W.E. Thirring., Ann. Phys. (N.Y.) 16, 96 (1961).

3. A.I. Nikishov, gr-qc/ 0710.4445 ,

4. L.D.Landau and E.M.Lifshitz, The classical theory of fields,

Addison-Wesley, Cambridge, MA, 1971).

5.J.Schwinger, Particles, Sources, and Fields. V.1 Addison-Wesley (1970).

6. S.Weinberg, Gravitation and Cosmology, New York (1972).

7. C.W.Misner, K.S.Thorne, J.A.Wheeler, Gravitation. San Francisco(1973). 